\title{
Gastric mucin phenotype indicates aggressive biological behaviour in early differentiated gastric adenocarcinomas following endoscopic treatment
}

Kai Song, Qi Yang, Yu Yan, Xiaoyan Yu, Kanlun Xu and Jinghong Xu*

\begin{abstract}
Background: The distribution of mucin phenotypes and their relationship with clinicopathological features in early differentiated gastric adenocarcinomas in a Chinese cohort are unknown. We aimed to investigate mucin phenotypes and analyse the relationship between mucin phenotypes and clinicopathological features, especially biological behaviours, in early differentiated gastric adenocarcinomas from endoscopic specimens in a Chinese cohort.

Methods: Immunohistochemical staining of CD10, MUC2, MUC5AC, and MUC6 was performed in 257 tissue samples from patients with early differentiated gastric adenocarcinomas. The tumour location, gross type, tumour size, histological type, depth of invasion, lymphovascular invasion, mucosal background and other clinicopathological parameters were evaluated. The relationship between mucin phenotypes and clinicopathological features was analysed with the chi-square test.

Results: The incidences of gastric, gastrointestinal, intestinal and null phenotypes were $21 \%, 56 \%, 20$ and 3\%, respectively. The mucin phenotypes were related to histology classification $(P<0.05)$. The proportion of the gastric phenotype became greater during the transition from differentiated to undifferentiated $(P<0.05)$. Complete intestinal metaplasia was higher in the gastric and intestinal phenotypes than in the gastrointestinal phenotype $(P<0.05)$. Tumours with poorly differentiated adenocarcinoma were mainly of the gastric phenotype, which was significantly higher than that of purely differentiated tubular adenocarcinoma $(P<0.05)$, and the depth of invasion in the mixed type was deeper $(P<0.05)$. Neither recurrence nor metastasis was detected.

Conclusions: The mucin phenotype of early-differentiated gastric adenocarcinoma has clinical implications, and the gastric phenotype has aggressive biological behaviour in early differentiated gastric cancers, especially in those with poorly differentiated adenocarcinoma or papillary adenocarcinoma components.
\end{abstract}

Keywords: Gastric cancer, Early stage, Mucin core protein, Gastric Mucin phenotype, Biological behaviour

\footnotetext{
* Correspondence: zydxjh@zju.edu.cn

Department of Pathology, The Second Affiliated Hospital, Zhejiang

University School of Medicine, Hangzhou, China
}

(c) The Author(s). 2021 Open Access This article is licensed under a Creative Commons Attribution 4.0 International License, which permits use, sharing, adaptation, distribution and reproduction in any medium or format, as long as you give appropriate credit to the original author(s) and the source, provide a link to the Creative Commons licence, and indicate if changes were made. The images or other third party material in this article are included in the article's Creative Commons licence, unless indicated otherwise in a credit line to the material. If material is not included in the article's Creative Commons licence and your intended use is not permitted by statutory regulation or exceeds the permitted use, you will need to obtain permission directly from the copyright holder. To view a copy of this licence, visit http://creativecommons.org/licenses/by/4.0/ The Creative Commons Public Domain Dedication waiver (http://creativecommons.org/publicdomain/zero/1.0/) applies to the data made available in this article, unless otherwise stated in a credit line to the data. 


\section{Background}

Gastric cancer (GC), one of the most common human cancers worldwide, is a disease with multiple pathogenic factors, various prognoses and different responses to treatments. Thus, properly distinguishing those with worse prognoses from those with better prognoses appears to be significantly important. Four different morphology -based classification systems exist, the World Health Organization (WHO/2019) [1], the Japanese Gastric Cancer Association (JGCA/2017) [1], Laurén [2] and Nakamura [3]. According to the WHO classification, GCs are subclassified into papillary, tubular, poorly cohesive, mucinous and mixed types. In the JGCA classification, the subtypes are papillary (pap), tubular (tub), poorly differentiated (por), signet-ring cell (sig), and mucinous (muc), which are similar to the subtypes used by the WHO. GCs are divided into intestinal and diffuse types using Laurén's classification or into differentiated and undifferentiated types based on Nakamura's classification [2-4]. The differentiated type contains pap, tub1, and tub2 according to the JGCA classification and papillary and well/moderately differentiated adenocarcinoma according to the WHO classification. These different histological types exhibit distinct biological behaviours.

The mucous produced by cancers is one of the factors determining the nature of biological behaviour. The main component of mucous is a high-molecular-weight glycoprotein called mucin [5]. As cancer progresses, the nature of the mucous changes relative to the degree of biological malignancy. In the $1990 \mathrm{~s}$, with the progress of structural analysis of mucin and the widespread use of monoclonal antibodies to the core protein of mucin, a mucin phenotype subclassification emerged. Mucin phenotype subclassification was entirely based on the mucin expression pattern, independent of histological features. Thus, GCs are classified into gastric, intestinal, gastrointestinal and null mucin phenotypes [4-6]. Previous studies have reported that the gastric phenotype has a higher potential for invasion and metastasis than the intestinal type, which results in a worse prognosis of GCs [7-11]. However, studies have mostly focused on advanced gastric cancers, and early gastric cancers are rarely investigated.

Early gastric cancer (EGC) is defined as tumour invasion confined to the mucosa and submucosa, irrespective of regional lymph node metastasis [12]. Endoscopic mucosal resection (EMR) and endoscopic submucosal dissection (ESD) are used as treatments for some intramucosal carcinomas and submucosal lesions, which have a very low probability of lymph node metastasis $[13,14]$.

To our knowledge, there is no research exploring biological role of mucin phenotypes in EGCs using EMR/
ESD by Chinese investigators. Little information is available on the effects of mucin phenotypes on the clinicopathological features of EGCs in a Chinese cohort. Accordingly, we examined mucin expression and mucin phenotypes and explored mucin phenotype clinicopathological characteristics and biological behaviour.

\section{Methods}

\section{Patients and tissue specimens}

Our study consisted of 257 consecutive patients who underwent EMR/ESD for differentiated EGCs between January 2012 and June 2018 at The Second Affiliated Hospital of Zhejiang University, China. The group comprised 182 men and 75 women with an age range of 29 to 87 (mean 64) years old.

The location of each lesion was classified in terms of the upper (27 cases), middle (46 cases) and lower (184 cases) thirds of the stomach. The size of each lesion was measured by the maximum diameter, which ranged from 0.1 to 6.5 (mean 1.5) centimetres. The protruding category (52 cases) included type 0-I and 0-IIa, the depressed (102 cases) category contained type 0-IIc and III, and all the other cases were considered under the protruding and depressed category (103 cases). Based on the WHO and JGCA classification, the EGCs were subclassified into well differentiated tubular adenocarcinoma (well-diff/tub 1, 198 cases), moderately differentiated tubular adenocarcinoma (mod-diff/tub 2, 37 cases), papillary adenocarcinoma (3 cases) and mixed (tub-pap/sig/por, 19 cases). In the mixed cases, the undifferentiated components (sig/por) were less than $50 \%$.

\section{Immunohistochemistry}

All specimens were fixed with $10 \%$ buffered formalin, embedded in paraffin, cut into $4-\mu \mathrm{m}$-thick sections, and subjected to haematoxylin and eosin (HE) staining. MUC2 was detected by mAb Ccp58 (Zsbio, 1:100), MUC5AC by mAb MRQ-19 (Zsbio, 1:100), MUC6 by mAb MRQ-20 (Zsbio, 1:100), and CD10 (Zsbio, 1:100) by immunohistochemistry (IHC). IHC was performed by using the Ventana NexES Staining System (Roche, Benchmark $\left.{ }^{\circ} \mathrm{XT}\right)$. The marker CD10 exhibited both cytoplasmic and glandular luminal reactivity, whereas MUC5AC, MUC6 and MUC2 exhibited only cytoplasmic reactivity. The staining results were categorized as positive when at least one single cell among the carcinoma cells was stained and negative when none of the carcinoma cells were stained [6].

\section{Classification of mucin phenotype}

Based on MUC5AC, MUC6, MUC2 and CD10, EGCs can be classified into the gastric phenotype (G-type), gastrointestinal phenotype (GI-type), intestinal phenotype (I-type) and null phenotype (N-type) [4, 6, 15-17]. 
The following criteria were used for the classification of mucin phenotypes: (1) the G-type shows positive staining for at least one of the MUC5AC and MUC6, while CD10 and MUC2 are both negative; (2) the I-type shows positive staining for $\mathrm{CD} 10$ and/or MUC2, while MUC5AC and MUC6 are both negative; (3) the GI-type shows positive staining for marker CD10 and/or MUC2 associated with one of the markers MUC5AC and MUC6 positive; and(4) if none of the four markers are positive, the phenotype is classified as N-type.

In addition, among the GI-types, we labelled one expressing more MUC5AC and/or MUC6 than MUC2 and CD10 as a gastric-predominant GI phenotype (GI-G type); otherwise, we labelled it as an intestinalpredominant phenotype (GI-I type).

\section{Statistical analysis}

Associations between mucin expression profiles and clinicopathological parameters were examined by the chi-square test or Fisher's exact test. Statistical significance was established to be $P<0.05$. Statistical calculations were performed with IBM SPSS Statistics (version 23.0).

\section{Results}

\section{Expression of mucin markers and mucin phenotype in}

\section{early gastric cancers}

The expression percentages of CD10, MUC2, MUC5AC and MUC6 in all EGCs were $43.58 \%(112 / 257), 63.81 \%$ (164/257), $64.98 \%(167 / 257)$ and $72.76 \%(187 / 257)$ respectively (Fig. 1).

Two hundred fifty-seven EGCs were classified as Gtype $(21 \%, 54 / 257)$, GI-type $(56 \%, 144 / 257)$, I-type (20\%, 51/257) and N-type (3\%, 8/257). The GI-type contained the GI-G type $(72 \%, 103 / 144)$ and GI-I type
(28\%, 41/144). There were more cases of G-type and GI-G type $(61 \%, 157 / 257)$ than I-type and GI-I type (36\%, 95/257) (Fig. 2).

\section{Relationship between mucin phenotype and clinicopathological features}

The relationship between mucin phenotype and clinicopathological features is summarized in Table 1. The mucin phenotypes were significantly related to the JGCA and WHO classifications $(P<0.05)$, but the parameters of sex, age, margin, colour, tumour size, gross type, depth of invasion, and lymphovascular invasion did not significantly differ among those mucin phenotypes $(P>$ $0.05)$. The I-type had the highest proportion of differentiated EGCs among the four mucin phenotypes (100.0\% vs. $79.7 \%$ vs. $83.1 \%$ vs. $87.5 \%, P=0.027)$. The G-type group had a higher proportion of tub-por/sig and pap/ tub-pap cases than the I-, GI- and N-type groups (20.4\% vs. $7.0 \%$ vs. $0.0 \%$ vs. $12.5 \%, P=0.027$ ) according to the JGCA classification. According to the WHO classification, the G-type had more mixed histological components $(18.5 \%$ vs. $5.6 \%$ vs. $0.0 \%$ vs. $12.5 \%, P=0.006)$ than the other mucin phenotypes.

\section{Relationship between mucin phenotypes and background mucosa}

Intestinal metaplasia (IM) of background mucosa was observed in 199 of 249 (79.9\%) cases (G-, GI- and GItype), including 38 cases of incomplete IM and 161 cases of complete IM. IM did not significantly differ among mucin phenotypes $(P>0.05)$. However, the presence of incomplete and complete IM was significantly different in distinct mucin phenotypes $(P=0.004, P=0.018)$. The presence of incomplete IM in GI-type EGCs was higher than that in the G-type and I-type EGCs $(21.5 \%$ vs.

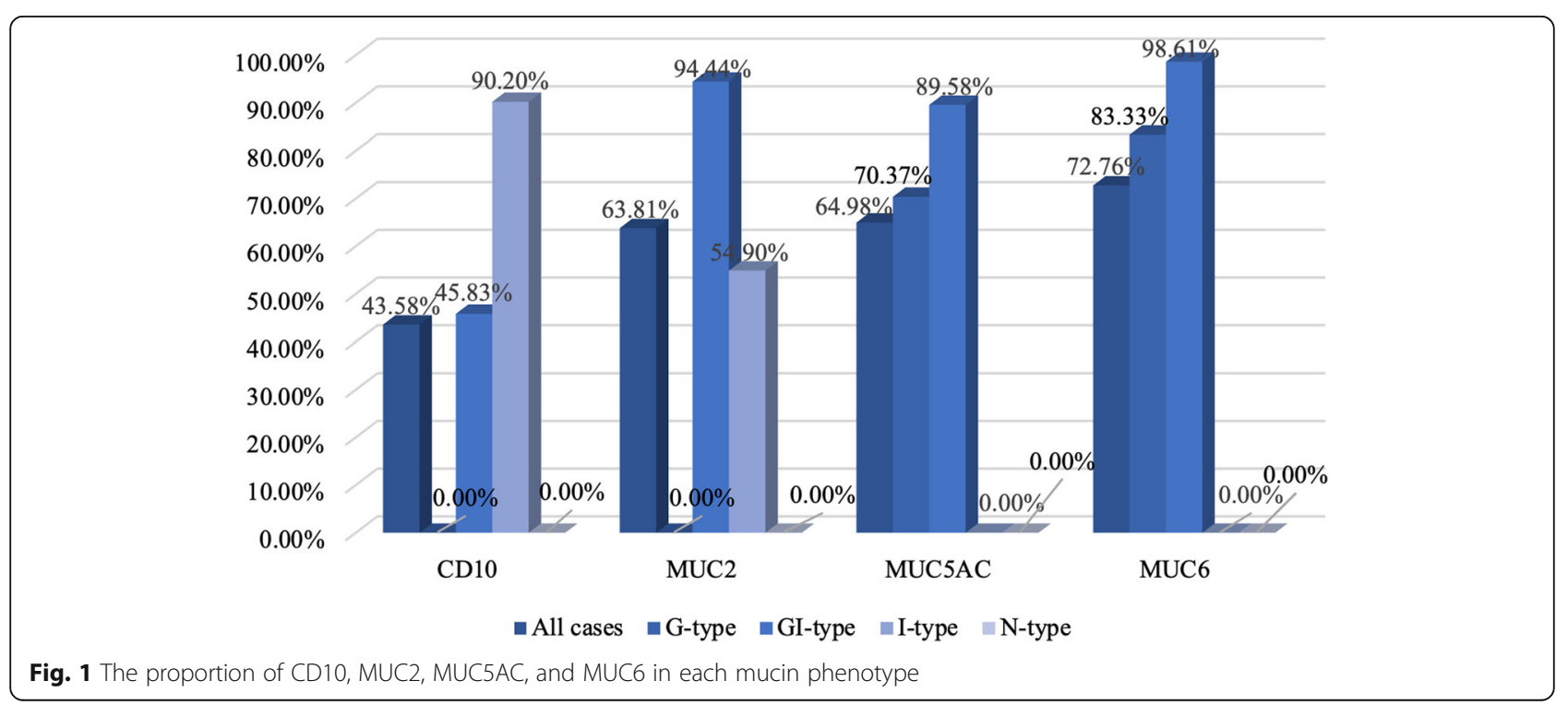




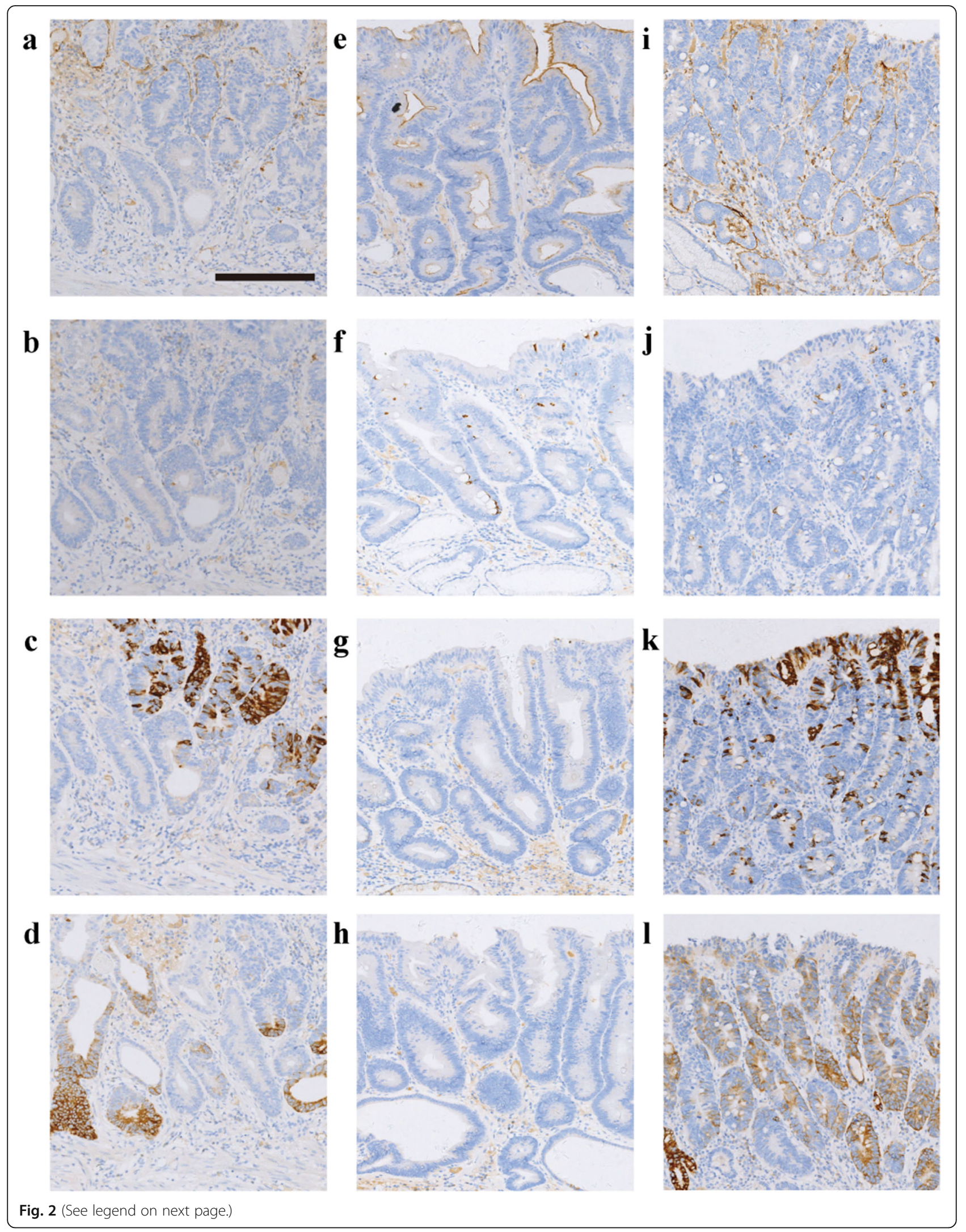


(See figure on previous page.)

Fig. $2 \mathrm{HC}$ staining of the gastric phenotype, intestinal phenotype and gastrointestinal phenotype. G-type (a-d) shows negative staining for CD10 (a) and MUC2 (b) and positive staining for MUC5AC (c) and MUC6 (d). In contrast, l-type (e-h) shows CD10 (e) and MUC2 (f) marker positivity and negative staining for MUC5AC (g) and MUC6 (h) negative. Gl-type (i-I) shows positive staining for CD10 (i), MUC2 (j),MUC5AC (k) and MUC6 (I). (Scale bar $=200 \mu \mathrm{m}, \mathrm{HE}$ and $\mathrm{HC} \times 10$ )

$9.3 \%$ vs. $3.9 \%)$. In the contrast, $77.8 \%(42 / 54)$ of G-type EGCs and $70.6 \%(36 / 51)$ of I-type EGCs exhibited complete IM, which was higher than the $57.6 \%(83 / 114)$ of GI-type EGCs. The IM status of the background mucosa and the relationship with mucin phenotypes are shown in Table 2.

\section{Biological behaviour of mucin phenotypes}

In addition to tubular adenocarcinoma components, 22 of the 257 cases also contained components of papillary adenocarcinoma, poorly differentiated carcinoma, or signet ring cell carcinoma. Fifteen cases $(68.18 \%)$ contained por/sig components, and the other 7 (31.82\%) contained pap components. Eleven cases showed the G-type, 10 cases showed the GI-type, only one case showed the Ntype, and none of them showed the I-type. Among the 10 GI-type cases, 9 cases showed the GI-G type, and one showed the GI-I type. Almost all the 22 patients showed the G- and GI-G types, which was significantly higher than the number of I-type patients $(P=0.011)$. In addition, the 22 patients had a higher proportion of infiltration into the submucosa $(P<0.001)$. (Table 3 ; Figs. 3 and 4).

\section{Follow-up}

Six patients underwent additional gastrectomy, and there was no residual tumour or lymph node metastasis. All patients were under close follow-up, and neither recurrence nor metastasis was detected.

\section{Discussion}

The mucin phenotype classification is based on the mucin marker expression profile. After year 2000, the gastric and intestinal mucin phenotypes were analysed by IHC [15]. The mucin markers MUC5AC, MUC6, MUC2 and CD10 were considered necessary, although there is no consensus on the number of markers that should be used to define a mucin phenotype or the percentage of tumour cells that must be stained $[6-8,11$, $15,18]$. MUC5AC is a secreted mucin expressed in the surface mucous epithelium of normal gastric mucosa. High expression of MUC6 is observed in fundic mucous neck cells and pyloric glands of gastric mucosa. CD10 is a marker for the brush border on the luminal surface of the small intestine. In the normal adult intestine, MUC2 expression is observed in the perinuclear areas of goblet cells.
We showed that the expression of MUC5AC, MUC6, MUC2 and CD10 was detected in 167 (64.98\%), 187 (72.76\%), 164 (63.81\%), and $112(43.58 \%)$ of the 257 EGCs, respectively. In previous studies, the expression percentages of MUC5AC, MUC6, MUC2 and CD10 in GCs were 55.1-67.5\%, 44.9-64\%, 35.4-49.3\% and 20.6$20.9 \%$, respectively $[19,20]$, and for EGCs, the expression of each mucin marker was 68.75-96.8\%, 19.6$71.58 \%, 25-62.10 \%$, and 0-79\%, respectively [6, 11, 21].

Based on the combinations of expression of these markers, the 257 EGCs were classified into the G-type (21\%, 54/257), GI-type (56\%, 144/257), I-type (20\%, 51/ 257 ) and N-type (3\%,8/257); in previous reports, the incidence percentages of each of these mucin phenotypes were found to be $15-41.1 \%, 20.3-60.1 \%, 18.5-46.6 \%$, and $3.7-31.6 \%$, respectively, in advanced GCs [3, 13, 14], and $7.9-36.8 \%, 18.8-41.2 \%, 15.4-55.56 \%$, and $0-11.1 \%$, respectively, in early- stage GCs [7, 11, 19-25]. Our results were consistent with these studies. The reported expression ranges vary greatly among different investigators, and different markers, antibodies and case groups may account for this discrepancy. Koyama et al. reported that the incidence of G-type was $19.3 \%$ [26], which was similar to that found in the present study (21\%); however, in his report, the incidence of I-type was much higher than that of G-type ( $43.8 \%$ vs. $19.3 \%)$, as was reported by Fabio et al. [11]. While Tajima et al. [22] reported the opposite result, since in their study, the incidence of the G-type was much higher than that of the I-type ( $36.8 \%$ vs. $15.4 \%$ ). In our study, the incidence of G-type was almost the same as that of I-type (21\% vs. $20 \%)$. Overall, based on our data, much more than half of these cases were classified as G- and GI-G type GC (61.09\%, 157/257), which is much higher than the incidence of I- and GI-I type GC (36.96\%,95/257). A previous report revealed that almost all intramucosal GC cases exhibited the gastric phenotype, including the GI phenotype [15].

The relationship between mucin phenotypes and clinicopathological features was investigated. We found that histology classification (both the JGCA and WHO classification) was closely related to the mucin phenotype. The incidence of I-type was greater than those of the G-, GI- and N-type (100.0\% vs. $79.7 \%, 93.1 \%, 87.5 \%)$ in differentiated tubular adenocarcinoma. The G-type was histologically significantly correlated with the mixed type (with poorly differentiated/papillary carcinoma). Our data showed that the proportion of G-type carcinoma 
Table 1 Relationship between Mucin Phenotypes and Clinicopathological Features

\begin{tabular}{|c|c|c|c|c|c|c|}
\hline & G-type & Gl-type & I-type & N-type & $x^{2}$ value & $P$ value \\
\hline Sex & & & & & 0.671 & 0.906 \\
\hline Male & $40(74.1 \%)$ & $99(68.8 \%)$ & $37(72.5 \%)$ & $6(75.0 \%)$ & & \\
\hline Female & $14(25.9 \%)$ & $45(31.2 \%)$ & $14(27.5 \%)$ & $2(25.0 \%)$ & & \\
\hline Age & & & & & 0.412 & 0.945 \\
\hline$\leq 64$ years & $28(51.9 \%)$ & $75(52.1 \%)$ & $29(56.9 \%)$ & $4(50.0 \%)$ & & \\
\hline$>64$ years & $26(48.1 \%)$ & $69(47.9 \%)$ & $22(43.1 \%)$ & 4(50.0\%) & & \\
\hline Tumor Size & & & & & 2.356 & 0.502 \\
\hline$\leq 2 \mathrm{~cm}$ & $34(63.0 \%)$ & $99(68.8 \%)$ & $32(62.7 \%)$ & 7(87.5 \%) & & \\
\hline$>2 \mathrm{~cm}$ & $20(37.0 \%)$ & $45(31.2 \%)$ & 19(37.3\%) & $1(12.5 \%)$ & & \\
\hline Margin & & & & & 4.269 & 0.224 \\
\hline Distinct & $35(64.8 \%)$ & $111(77.1 \%)$ & $40(78.4 \%)$ & $5(62.5 \%)$ & & \\
\hline Indistinct & 19(35.2\%) & $33(22.9 \%)$ & $11(21.6 \%)$ & $3(37.5 \%)$ & & \\
\hline Color & & & & & 3.423 & 0.337 \\
\hline Darken & $31(57.4 \%)$ & $75(52.1 \%)$ & $34(66.7 \%)$ & $5(62.5 \%)$ & & \\
\hline Faded & $23(42.6 \%)$ & $69(47.9 \%)$ & 17(33.3\%) & $3(37.5 \%)$ & & \\
\hline Tumor Location & & & & & 5.819 & 0.407 \\
\hline Upper & $4(7.4 \%)$ & $15(10.4 \%)$ & $8(15.7 \%)$ & $0(0.0 \%)$ & & \\
\hline Middle & $12(22.2 \%)$ & $21(14.6 \%)$ & $12(23.5 \%)$ & $1(12.5 \%)$ & & \\
\hline Low & $38(70.4 \%)$ & $108(75.0 \%)$ & $31(60.8 \%)$ & $7(87.5 \%)$ & & \\
\hline Tumor Location & & & & & 0.787 & 0.842 \\
\hline EGJ & $4(7.4 \%)$ & $7(4.9 \%)$ & $3(5.9 \%)$ & $0(0.0 \%)$ & & \\
\hline No-EGJ & $50(92.6 \%)$ & 137(95.1\%) & 48(94.1\%) & $8(100.0 \%)$ & & \\
\hline Gross type & & & & & 8.943 & 0.162 \\
\hline Protruding & $10(18.5 \%)$ & $32(22.2 \%)$ & 10(19.6\%) & $0(0.0 \%)$ & & \\
\hline Depressed & $16(29.6 \%)$ & $57(39.6 \%)$ & $26(51.0 \%)$ & $3(37.5 \%)$ & & \\
\hline Protruding-Depressed & $28(51.9 \%)$ & $55(38.2 \%)$ & $15(29.4 \%)$ & $5(62.5 \%)$ & & \\
\hline JGCA Classification & & & & & 16.870 & 0.027 \\
\hline tub1 & $34(62.9 \%)$ & $112(77.8 \%)$ & $45(88.2 \%)$ & $7(87.5 \%)$ & & \\
\hline tub2 & $9(16.7 \%)$ & $22(15.2 \%)$ & $6(11.8 \%)$ & $0(0.0 \%)$ & & \\
\hline tub-por/sig & $7(13.0 \%)$ & $7(4.9 \%)$ & $0(0.0 \%)$ & $1(12.5 \%)$ & & \\
\hline pap/tub-pap & $4(7.4 \%)$ & $3(2.1 \%)$ & $0(0.0 \%)$ & $0(0.0 \%)$ & & \\
\hline WHO Classification & & & & & 18.052 & 0.017 \\
\hline Tubular, well-diff & $34(62.9 \%)$ & $112(77.8 \%)$ & $45(88.2 \%)$ & $7(87.5 \%)$ & & \\
\hline Tubular, mod-diff & $9(16.7 \%)$ & $22(15.2 \%)$ & $6(11.8 \%)$ & $0(0.0 \%)$ & & \\
\hline Papillary & $1(1.9 \%)$ & $2(1.4 \%)$ & $0(0.0 \%)$ & $0(0.0 \%)$ & & \\
\hline Mixed & $10(18.5 \%)$ & $8(7.0 \%)$ & $0(0.0 \%)$ & $1(12.5 \%)$ & & \\
\hline Depth of Invasion & & & & & 1.360 & 0.681 \\
\hline M & $47(87.0 \%)$ & 132(91.7\%) & $46(90.2 \%)$ & $8(100.0 \%)$ & & \\
\hline SM & $7(13.0 \%)$ & $12(8.3 \%)$ & $5(9.8 \%)$ & $0(0.0 \%)$ & & \\
\hline Lymphovascular invasion & & & & & 2.541 & 0.687 \\
\hline$(+)$ & $1(1.9 \%)$ & $1(0.7 \%)$ & $0(0.0 \%)$ & $0(0.0 \%)$ & & \\
\hline$(-)$ & $53(98.1 \%)$ & 143(99.3\%) & $51(100.0 \%)$ & $8(100.0 \%)$ & & \\
\hline
\end{tabular}

+ , present; - absent

EGJ esophagogastric junction, well-diff well differentiated, mod-diff moderately differentiated, $M$ mucosa, SM submucosa 
Table 2 Relationship between mucin phenotypes and background mucosa

\begin{tabular}{|c|c|c|c|c|c|}
\hline & G-type & Gl-type & I-type & $x^{2}$ value & $P$ value \\
\hline Intestinal metaplasia & & & & 2.685 & 0.265 \\
\hline$(+)$ & $47(87.1 \%)$ & $114(79.2 \%)$ & $38(74.5 \%)$ & & \\
\hline$(-)$ & $7(12.9 \%)$ & $30(20.8 \%)$ & $13(25.5 \%)$ & & \\
\hline Incomplete intestinal metaplasia & & & & 10.948 & 0.004 \\
\hline$(+)$ & $5(9.3 \%)$ & $31(21.5 \%)$ & $2(3.9 \%)$ & & \\
\hline$(-)$ & 49(90.7\%) & $113(78.5 \%)$ & 49(96.1\%) & & \\
\hline Complete intestinal metaplasia & & & & 7.957 & 0.018 \\
\hline$(+)$ & $42(77.8 \%)$ & $83(57.6 \%)$ & $36(70.6 \%)$ & & \\
\hline$(-)$ & $12(22.2 \%)$ & $61(42.4 \%)$ & $15(29.4 \%)$ & & \\
\hline
\end{tabular}

+, present; - absent

increased during the transition from solely differentiated type to mixed type carcinoma. Mixed-type early-stage carcinoma more frequently expressed G-type mucin, and Gtype tumours were associated with a higher rate of undifferentiated-type tumours than I-type tumours [7, 22].

There were no significant differences between mucin phenotypes and other parameters, including sex, age, margin, colour, tumour size, gross type, depth of invasion, and lymphovascular invasion $(P>$ $0.05)$. These results are consistent with those of other studies in the literatures [22, 24, 27], and there was no clear correlation between phenotypes and clinicopathological characteristics, including sex, age, tumour size, location, macroscopic features, lymphatic or venous invasion, or lymph node metastasis in the case of the differentiated type [22, 24, 27]. Koseki et al. [7] and Oya et al. [28] reported that the incidence of lymphatic invasion, venous invasion and lymph node metastasis in gastric phenotype carcinomas was significantly higher than that in intestinal phenotype carcinomas. In addition, G-type EGCs were correlated with some distinct macroscopic features, namely, a smaller tumour diameter [15], discoloured surface and non-wavy tumour margins [23, 29]. G-type differentiated adenocarcinomas showed a depressed type, indistinct margins and monotonous colour tone across the mucosal layer, whereas I-type adenocarcinomas had an elevated, distinct margin and a red mucosa $[3,4$, 30]. The discrepancy of these results may have been due to heterogeneous components that contained poorly differentiated adenocarcinoma [27].

Intestinal metaplasia has been frequently observed surrounding GC, especially differentiated adenocarcinomas. IM has malignant potential and has been regarded as a precursor of gastric neoplasms. According to Laurén, intestinal-type adenocarcinoma is preceded by metaplastic changes, while diffuse-type adenocarcinoma arises in non-IM gastric mucosa [2]. In the current study, background mucosal IM was observed in $79.9 \%$ of cases among the G-, GI- and Itype EGCs and $87.0 \%$ of cases among the G-type EGCs. $25 \%$ of I-type cases arose from the normal mucosa without IM. IM did not significantly differ among mucin phenotypes $(P>0.05)$. However, incomplete and complete IM significantly differed with respect to mucin phenotypes $(P=0.004, P=0,018)$. A total of $77.78 \%(42 / 54)$ of G-type and $70.6 \%(36 / 51)$ of I-type patients had complete IM, which was higher than the rates among GI-type patients (83/114, $57.6 \%)$. The expression of incomplete IM in GI-type EGCs was higher than in G- and I-type EGCs $(21.5 \%$ vs. $9.3 \%$ vs. $3.9 \%$ ). Our results demonstrated a

Table 3 Relationship between Biological Behavior and Mucin Phenotypes

\begin{tabular}{|c|c|c|c|c|c|}
\hline & tub & tub-por/sig & pap/tub-pap & $x^{2}$ value & $P$ value \\
\hline Depth of Invasion & & & & 15.824 & 0.000 \\
\hline M & 219(93.2\%) & $9(60.0 \%)$ & $5(71.4 \%)$ & & \\
\hline SM & 16(6.8\%) & $6(40.0 \%)$ & $2(28.6 \%)$ & & \\
\hline Mucin Phenotype & & & & 14.743 & 0.011 \\
\hline G-type & $43(18.3 \%)$ & $7(46.7 \%)$ & $4(57.1 \%)$ & & \\
\hline Gl-type & $134(57.0 \%)$ & $7(46.7 \%)$ & $3(42.9 \%)$ & & \\
\hline I-type & $51(21.7 \%)$ & $0(0.0 \%)$ & $0(0.0 \%)$ & & \\
\hline N-type & $7(3.0 \%)$ & $1(6.6 \%)$ & $0(0.0 \%)$ & & \\
\hline
\end{tabular}

$M$ mucosa, SM submucosa 

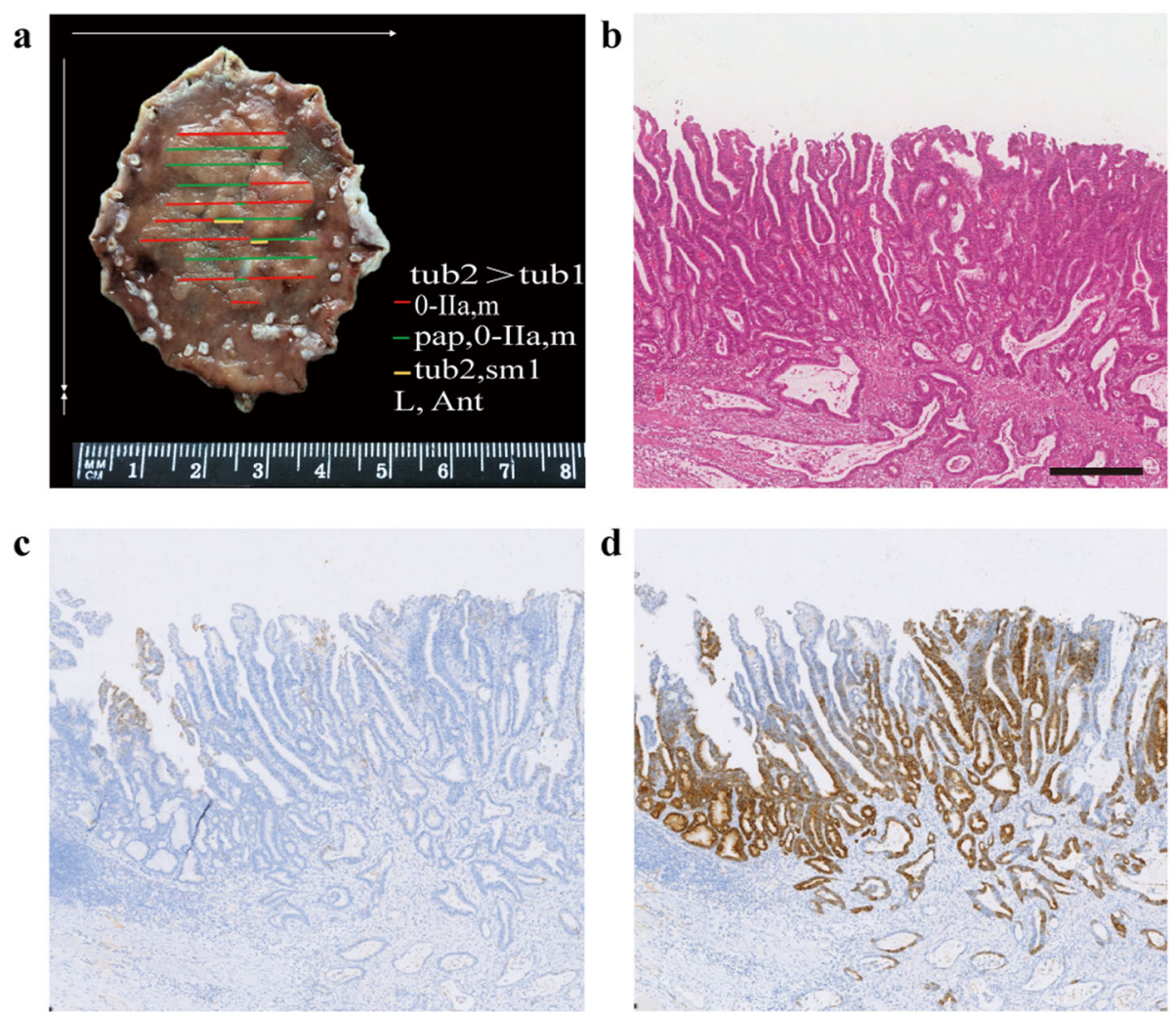

Fig. 3 Tubular adenocarcinoma mixed with papillary adenocarcinoma showing the gastric mucin phenotype. Reconstructive map (a). H\&E staining showing papillary structure on the surface and tubular adenocarcinoma in the submucosa; invasive depth is $300 \mu \mathrm{m}$ (b). Focal positive staining for MUC5AC (c) and strongly positive staining for MUC6 (d), invasive tubular adenocarcinoma showing MUC5AC negative (c) and MUC6 positive (d) status. (Scale bar $=400 \mu \mathrm{m}, \mathrm{HE}$ and $\mathrm{IHC} \times 10)$

remarkable difference between mucin phenotypes and the background mucosa. Similar results have been reported by Kabashima et al. [31] and Matsuoka [23]. The mucin phenotype of the carcinoma was independent of mucin phenotypic changes in the surrounding mucosa, and the carcinoma may undergo individual intestinalization. The G-type may imitate the surrounding mucosa, and the carcinomas and the background mucosa have an unstable status, as they commonly possess the hybrid phenotype of the stomach and the small intestine [23, 31].

Mucin phenotypes can indicate biological behaviour in GCs. G-type GCs have increased potential for invasion and metastasis due to infiltrating of deeper layers or more surrounding structures, a higher rate of lymph node metastasis, and poorer prognosis $[3,12$, 18, 21]. Even differentiated adenocarcinomas of the G-type had similar outcomes, focused on prognoses, as undifferentiated adenocarcinomas [7-10]. In our research, six patients underwent additional gastrectomy, and there was no residual tumour or lymph node metastasis. All patients were under close followup, and neither recurrence nor metastasis was detected. The mixed type (mixed with poorly differentiated or papillary adenocarcinoma) was mainly of the G-type, which was significantly higher than that of purely differentiated tubular adenocarcinoma $(P<$ $0.05)$, and the depth of infiltration was deeper $(P<$ $0.05)$. The G-type group had the highest proportion $(11 / 54,20.37 \%)$ with poorly differentiated/undifferentiated components, and almost all of them (19/22, $86.36 \%)$ expressed the G- and GI-G types. The mixed type may represent a progressive loss of glandular structure during progression of the cancer from the mucosa to advanced stage, and those with submucosal invasion was a risk factor for lymph node metastasis [7, 22, 32]. Differentiated EGC of G-type frequently changed histologically into signet ring-cell carcinoma or poorly differentiated adenocarcinoma. These results may imply more aggressive biological behaviour and poorer prognosis.

\section{Conclusions}

Our study reports the expression of mucin markers (MUC5AC, MUC6, MUC2 and CD10) and mucin phenotypes in differentiated EGC samples from ESD/EMR 

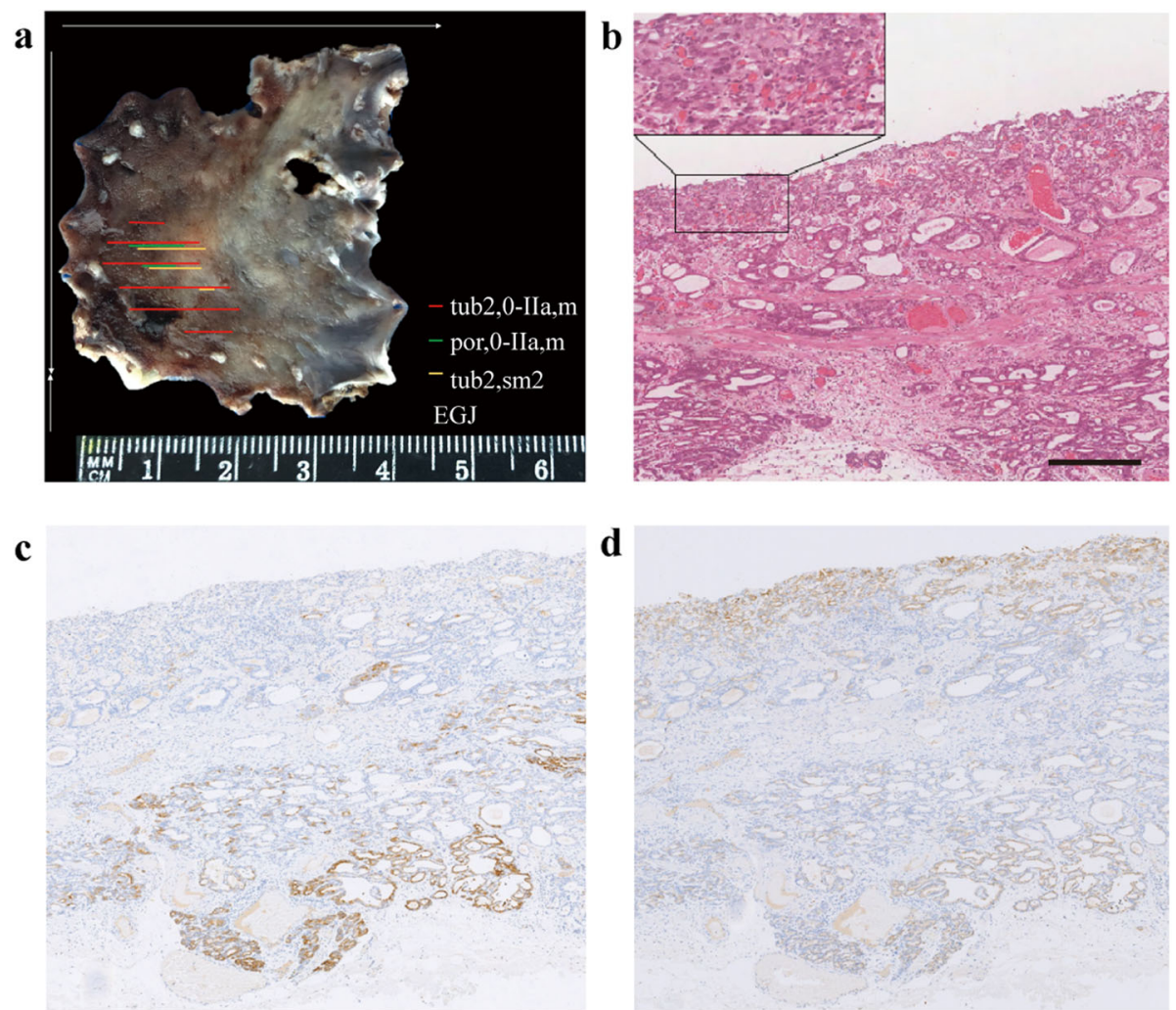

Fig. 4 Tubular adenocarcinoma mixed with poorly differentiated carcinoma showing the gastric mucin phenotype. Reconstructive map (a). H\&E staining showing poorly differentiated carcinoma in the mucosa and tubular adenocarcinoma in the submucosa; the invasive depth is $2500 \mu \mathrm{m}$ (b). Positive staining for MUC5AC (c) and MUC6 (d), and invasive tubular adenocarcinoma showing MUC5AC (c) and MUC6 (d) positivity. (Scale bar $=400 \mu \mathrm{m}, \mathrm{HE}$ and $\mathrm{IHC} \times 10)$

in the Chinese population. Mucin phenotypes of early differentiated gastric cancer are of clinical significance, and G-type GC exhibits aggressive biological behaviour in early differentiated GCs, especially in those with poorly differentiated adenocarcinoma or papillary adenocarcinoma components.

\section{Abbreviations}

GC: Gastric cancer; EGC: Early gastric cancer; WHO: World Health Organization; JGCA: Japanese Gastric Cancer Associatio; EMR: Endoscopic mucosal resection; ESD: Endoscopic submucosal dissection; G-type: Gastricphenotype; GI-type: Gastrointestinal-phenotype; I-type: Intestinal-phenotype; N-type: Null-type; Gl-G type: Gastric-predominant Gl phenotype; GI-I type: Intestinal-predominant phenotype; IM: Intestinal metaplasia; pap: Papillary adenocarcinoma; tub: Tubular adenocarcinoma; well-diff/tub 1: Well differentiated tubular adenocarcinoma; mod-diff/tub 2: Moderately differentiated tubular adenocarcinoma; por: Poorly differentiated; sig: Signetring cell; muc: Mucinous

\section{Acknowledgements}

We are very greateful to Dr. Jianhua Luo from Westlake University for her contribution to the adjustment of the figure format.

\section{Authors' contributions}

Conception and design: Jinghong $\mathrm{Xu}$, Acquisition and analysis of data: Jinghong Xu, Kai Song, Qi Yang and Yu Yan, Drafting the manuscript and figures: Kai Song and Jinhong Xu, Pathology technical support: Xiaoyan Yu, Kanlun Xu. The author(s) read and approved the final manuscript.
Funding

None.

\section{Availability of data and materials}

The data sets used and/or analyzed during the current study are available from the corresponding author on reasonable request.

\section{Declarations}

\section{Ethics approval and consent to participate}

All study procedures were in accordance with the ethical standards of the responsible committee on human experimentation (institutional and national) and with the Helsinki Declaration of 1964 and later versions.

\section{Consent for publication}

All authors agree to publish.

\section{Competing interests}

The authors declare no personal, financial,or institutional interest in any of the materials described in this article.

Received: 10 April 2021 Accepted: 5 July 2021

Published online: 13 July 2021

\section{References}

1. Board WC. of TE. Digestive System Tumours Who Classification of Tumours5th Edition. 2019.

2. Pekka Lauren. The two histological main types of gastric carcinoma: diffuse and so-called intestinal-type carcinoma. an attempt at a histoclinical classification. Acta Pathol Microbiol Scand. 1965;64:31-49. 
3. Nakamura K, Sugano H, Takagi K. Carcinoma of the stomach in incipient phase: its histogenesis and histological appearances. Gan. 1968;59:251-8. PMID: 5726267.

4. Namikawa T, Hanazaki K. Mucin phenotype of gastric cancer and clinicopathology of gastric-type differentiated adenocarcinoma. World J Gastroenterol. 2010;16:4634-9.

5. Yonezawa S, Higashi M, Yamada N, Yokoyama S, Kitamoto S, Kitajima S, et al. Mucins in human neoplasms: Clinical pathology, gene expression and diagnostic application. Pathol Int. 2011;61:697-716.

6. Toki F, Takahashi A, Aihara R, Ogata K, Ando H, Ohno T, et al. Relationship between clinicopathological features and mucin phenotypes of advanced gastric adenocarcinoma. World J Gastroenterol. 2010;16:2764-70.

7. Koseki K, Takizawa T, Koike M, Ito M, Nihei Z, Sugihara K. Distinction of differentiated type early gastric carcinoma with gastric type mucin expression. Cancer. 2000;89:724-32.

8. Yamaguchi Y, Takahashi S. Endoscopic mucosal resection (EMR) of early gastric cancer: usefulness of aspiration EMR using a cap-fitted scope. Gastrointest Endosc. 1998;48:548-9.

9. Machado JC, Nogueira AM, Carneiro F, Reis CA, Sobrinho-Simões M. Gastric carcinoma exhibits distinct types of cell differentiation: an immunohistochemical study of trefoil peptides (TFF1 and TFF2) and mucins (MUC1, MUC2, MUC5AC, and MUC6). J Pathol. 2000;190:437-43.

10. Saito A, Shimoda T, Nakanishi Y, Ochiai A, Toda G. Histologic heterogeneity and mucin phenotypic expression in early gastric cancer. Pathol Int Australia. 2001;51:165-71.

11. Hondo FY, Kishi H, Safatle-Ribeiro AV, Pessorrusso FCS, Ribeirol RU, MalufFilho F. Characterization of the Mucin Phenotype Can Predict Gastric Cancer Recurrence After Endoscopic Mucosal Resection. Arq Gastroenterol. 2017;54: 308-14.

12. Japanese Gastric Cancer Association. Japanese Classification of Gastric Carcinoma - 3rd English Edition. Gastric Cancer. 2011;14:101-12.

13. Gotoda T, Yanagisawa A, Sasako M, Ono H, Nakanishi Y, Shimoda T, et al. Incidence of lymph node metastasis from early gastric cancer: Estimation with a large number of cases at two large centers. Gastric Cancer. 2000;3: 219-25.

14. Japanese Gastric Cancer Association. Japanese gastric cancer treatment guidelines 2018 (5th edition). Gastric Cancer. 2021;24:1-21.

15. Oue N, Sentani K, Sakamoto N, Yasui W. Clinicopathologic and molecular characteristics of gastric cancer showing gastric and intestinal mucin phenotype. Cancer Sci. 2015;106:951-8.

16. Mizoshita T, Tsukamoto T, Nakanishi H, Inada K, Ogasawara N, Joh T, et al. Expression of $C d \times 2$ and the phenotype of advanced gastric cancers: relationship with prognosis. J Cancer Res Clin Oncol Germany. 2003;129: 727-34.

17. Oue N, Mitani Y, Aung PP, Sakakura C, Takeshima Y, Kaneko M, et al. Expression and localization of Reg IV in human neoplastic and nonneoplastic tissues: Reg IV expression is associated with intestinal and neuroendocrine differentiation in gastric adenocarcinoma. J Pathol England. 2005;207:185-98.

18. Saito K, Shimoda T. The histogenesis and early invasion of gastric cancer. Acta Pathol Jpn Australia. 1986;36:1307-18.

19. Kim DH, Shin $\mathrm{N}$, Kim GH, Song GA, Jeon TY, Kim DH, et al. Mucin expression in gastric cancer: Reappraisal of its clinicopathologic and prognostic significance. Arch Pathol Lab Med. 2013;137:1047-53.

20. Tatematsu M, Ichinose M, Miki K, Hasegawa R, Kato T, Ito N. Gastric and intestinal phenotypic expression of human stomach cancers as revealed by pepsinogen immunohistochemistry and mucin histochemistry. Acta Pathol Jpn Australia. 1990;40:494-504. https://doi.org/10.1111/j.1440-1827.1990.tb01 591.x PMID: 2220396

21. Han JP, Hong SJ, Kim HK, Kim HS, Lee YN, Lee TH, et al. Expression of immunohistochemical markers according to histological type in patients with early gastric cancer. Scand J Gastroenterol. 2016;51: 60-6.

22. Tajima Y, Yamazaki K, Nishino N, Morohara K, Yamazaki T, Kaetsu T, et al. Gastric and intestinal phenotypic marker expression in gastric carcinomas and recurrence pattern after surgery - Immunohistochemical analysis of 213 lesions. Br J Cancer. 2004:91:1342-8.

23. Matsuoka M, Aizawa Y, Nagamata H, Sutoh S, Miyagawa Y, Torii A. Significance of the mucin phenotype of early gastric cancer. Jikeikai Med J. 2003;50:29-36.
24. Kabashima A, Yao T, Sugimachi K, Tsuneyoshi M. Relationship between biologic behavior and phenotypic expression in intramucosal gastric carcinomas. Hum Pathol. 2002;33:80-6.

25. Egashira Y. Mucin histochemical study of differentiated adenocarcinoma of stomach. Nihon Shokakibyo Gakkai Zasshi Japan. 1994;91:839-48.

26. Fuchs CS, Mayer RJ. Gastric carcinoma. N Engl J Med. 1995;333:32-41.

27. Nakamura T, Yao T, Kabashima A, Nishiyama K, Maehara Y, Tsuneyoshi M. Loss of phenotypic expression is related to tumour progression in early gastric differentiated adenocarcinoma. Histopathology. 2005;47:357-67.

28. ï» ¿Oya M, Yao T, Tsuneyoshi M. A correlation of intramural invasion with mucin histochemistry, and immunohistochemical expressions of oncogenerelated proteins in early gastric carcinoma. I To Cho (Stomach Intestine). 1997;32:31-9.

29. Shimono K. [Macroscopic characteristics of depressed type early gastric cancer special reference to their mucosal localizations]. Nihon Shokakibyo Gakkai Zasshi. 1995;92(5):846-54. Japanese. PMID: 7783376.

30. Miyazawa M, Matsuda M, Yano M, Hara Y, Arihara F, Horita Y, et al. Gastric adenocarcinoma of the fundic gland (chief cell-predominant type): A review of endoscopic and clinicopathological features. World J Gastroenterol. 2016: 22:10523-31.

31. Kabashima A, Yao T, Sugimachi K, Tsuneyoshi M. Gastric or intestinal phenotypic expression in the carcinomas and background mucosa of multiple early gastric carcinomas. Histopathology. 2000;37:513-22.

32. Hanaoka N, Tanabe S, Mikami T, Okayasu I, Saigenji K. Mixed-histologic-type submucosal invasive gastric cancer as a risk factor for lymph node metastasis: Feasibility of endoscopic submucosal dissection. Endoscopy. 2009;41:427-32.

\section{Publisher's Note}

Springer Nature remains neutral with regard to jurisdictional claims in published maps and institutional affiliations.
Ready to submit your research? Choose BMC and benefit from:

- fast, convenient online submission

- thorough peer review by experienced researchers in your field

- rapid publication on acceptance

- support for research data, including large and complex data types

- gold Open Access which fosters wider collaboration and increased citations

- maximum visibility for your research: over $100 \mathrm{M}$ website views per year

At BMC, research is always in progress.

Learn more biomedcentral.com/submissions 